\title{
Controle Químico de Plantas AQuÁticas: Polygonum lapathifolium ${ }^{1}$
}

\author{
Chemical Control of Aquatic Plants: Polygonum lapathifolium
}

\begin{abstract}
TERRA M.A. ${ }^{2}$, NEGRISOLI, E. ${ }^{3}$, CARBONARI C.A. ${ }^{4}$, CARDOSO, L.R. ${ }^{3}$ e MARTINS, D. ${ }^{5}$
RESUMO - O objetivo deste trabalho foi avaliar a eficiência de alguns herbicidas no controle de Polygonum lapathifolium. O experimento foi instalado e conduzido no Núcleo de Pesquisas Avançadas em Matologia, pertencente à Faculdade de Ciências Agronômicas da UNESP Botucatu-SP. Plantas de P. lapathifolium foram coletadas no rio Tietê e cultivadas em caixas d'água de fibra de vidro de 0,6 x 0,6 x 0,45 m, contendo $15 \mathrm{~cm}$ de solo. Os tratamentos utilizados foram: 2,4-D amina a 720 a 1.440 g e.a. ha- ${ }^{-1}$ diquat a 480 g i.a. ha-1; glyphosate a 2.400 e 3.360 g e.a. ha ${ }^{-1}$ Aterbane $0,5 \%$ v/v; imazapyr a 250 e 500 g e.a. ha ${ }^{-1}$; e testemunha sem aplicação de herbicida. A aplicação foi realizada com um pulverizador costal pressurizado a $\mathrm{CO}_{2}$, a pressão constante de 2,0 bars, equipado com barra de pulverização munida de dois bicos de jato plano, tipo Teejet XR $110.02 \mathrm{VS}$. O consumo de calda foi de $200 \mathrm{~L} \mathrm{ha}^{-1}$. O delineamento experimental utilizado foi o inteiramente casualizado, com três repetições. As avaliações foram visuais, atribuindo-se notas para os sintomas de injúrias de acordo com uma escala percentual. Apenas o herbicida glyphosate, independentemente da dose utilizada, controlou as plantas de Polygonum lapathifolium, atingindo 100\% aos 46 dias após a aplicação. Inicialmente o herbicida diquat promoveu injúrias severas, entretanto, no decorrer do período de avaliações, elas se dissiparam e as plantas rebrotaram.
\end{abstract}

Palavras-chave: erva-de-bicho, glyphosate, diquat, 2,4-D, imazapyr.

\begin{abstract}
The objective of this trial was to study the efficacy of some herbicides in controlling P. lapathifolium. The experiment was carried out at Núcleo de Pesquisas Avançadas em Matologia of the Faculdade de Ciências Agronômicas/Botucatu - São Paulo, Brazil. Polygonum lapathifolium plants were collected in the Tietê River and cultivated in $0.6 \times 0.6 \times 0.45 \mathrm{~m}$ fiber glass tanks containing $15 \mathrm{~cm}$ of soil. The herbicides used were 2,4-D amine at 720 and $1,440 \mathrm{~g}$ e.a. ha $\mathrm{h}^{-1}$, diquat at $480 \mathrm{~g}$ a.i. $\mathrm{ha}^{-1}$, glyphosate at 2,400 and 3,360 g e.a. ha $\mathrm{g}^{-1}$ Aterbane $0.5 \% \mathrm{v} / \mathrm{v}$, imazapyr at 250 and $500 \mathrm{~g}$ i.a. ha $\mathrm{h}^{-1}$ and an untreated plot. The application was carried out using a backpack sprayer pressurized with $\mathrm{CO}_{2}$ at 2,O bar equipped with 2 flat fan nozzles type TeeJet XR110.02 VS. The application volume was $200 \mathrm{~L} \mathrm{ha}^{-1}$. The experiment was arranged in a completely randomized design, with 3 replications. Visual evaluations were conducted by grading the injury symptoms according to a percentage scale. The results showed that only glyphosate provided an excellent control of Polygonum lapathifolium even at the lowest rate, reaching 100\% at 46 days after application. Diquat caused severe injuries initially but these symptoms disappeared and the plants sprouted along the evaluation periods.
\end{abstract}

Key words: smartweed, glyphosate, diquat, 2,4-D, imazapyr.

\section{INTRODUÇÃO}

Os ambientes aquáticos, de forma geral, são formados por uma grande biodiversidade vegetal, que, em situação ecologicamente equilibrada, é essencial para a manutenção e o desenvolvimento desse ecossistema. Nesses ambientes, as plantas aquáticas são responsáveis pela oxigenação e depuração da água, servem de alimento para peixes e aves e

1 Recebido para publicação em 12.8.2002 e na forma revisada em 25.10.2002.

2 Aluno do curso de mestrado em Agricultura da Faculdade de Ciências Agronômicas - FCA/UNESP, 18603-970 Botucatu-SP; ${ }^{3}$ Aluno do curso de doutorado em Agricultura da FCA/UNESP; ${ }^{4}$ Aluno de graduação em Agronomia da FCA/ UNESP; ${ }^{5}$ Professor Doutor do Departamento de Produção Vegetal - FCA/UNESP. 
protegem as margens da ação erosiva da água (Tanaka, 1998).

A desestabilização desses locais pode ter como conseqüência, entre outras, um crescimento descontrolado de uma ou mais espécies vegetais, elevando sua população a níveis indesejáveis e prejudiciais, tornando necessária a adoção de medidas de controle.

As plantas aquáticas, na condição de daninhas, causam danos à geração de energia elétrica, à navegação, à captação de água, à atividade agropecuária, à atividade pesqueira, à saúde pública, ao lazer e turismo e ao ambiente (Tanaka, 1998). Deuber (1997) afirma que plantas aquáticas em excesso podem causar sérios prejuízos, reduzindo a disponibilidade e qualidade da água e gerando, assim, problemas ambientais, que podem ser relacionados com a saúde humana e animal. Outro problema é a presença macrófitas próximas a grandes centros urbanos, que possibilitam a procriação de mosquitos e outros vetores de doenças humanas, além de proporcionarem condições microaerofílicas com produção de substâncias de mau odor (Pitelli, 1998).

Polygonum lapathifolium, popularmente conhecida como erva-de-bicho, é uma espécie perene, pertencente à família Polygonaceae, que se desenvolve em ambientes aquáticos ou terrestres, propagando-se por sementes e principalmente por meio de secções de caule (Lorenzi, 2000). Na bacia do rio Tietê, no Estado de São Paulo, essas plantas encontram-se em situação de desequilíbrio, sendo citadas por Marcondes \& Tanaka (1997) como plantas de crescimento vigoroso nas margens de rios e lagos; com o bater das ondas, elas desprendemse do solo e passam a flutuar, formando ilhas gigantescas que continuam a desenvolver-se, oferecendo sérios riscos para a navegação e as hidrelétricas.

Estudos sobre o controle químico de plantas aquáticas no Brasil podem ser considerados recentes, porém Martins (1998) destaca que, em termos mundiais, é este método de controle o mais utilizado, através da aplicação de produtos como 2,4-D, glyphosate e diquat, endothal, compostos à base de cobre, fluridone e imazapyr.

Especificamente sobre o controle de P. lapathifolium, Natal \& Svetov (1973) comentam que o herbicida 2,4-D aplicado em mistura com dicamba proporcionou bom controle desta espécie. Whatley et al. (1984), por sua vez, afirmam que o herbicida glyphosate, quando aplicado em estágio inicial de desenvolvimento desta espécie, promove níveis de controle da ordem de $86 \%$.

Dessa forma, este trabalho teve como objetivo avaliar a eficiência de alguns herbicidas aplicados em diferentes doses no controle de Polygonum lapathifolium.

\section{MATERIAL E MÉTODOS}

O presente trabalho foi instalado e conduzido no NUPAM (Núcleo de Pesquisas Avançadas em Matologia), pertencente à Faculdade de Ciências Agrárias da UNESP, campus de Botucatu-SP.

Secções do caule de plantas de Polygonum lapathifolium, coletadas às margens do rio Tietê, foram plantadas e cultivadas em caixas de fibra de vidro com 0,60 x 0,60 m de largura por $0,45 \mathrm{~m}$ de profundidade, contendo $0,15 \mathrm{~m}$ de solo previamente adubado com $50 \mathrm{~g}$ de 4-14-8 e, em seguida, inundado.

As caixas foram alocadas em uma área coberta com brita no Núcleo, sendo dispostas a uma distância de 0,6 x 1,2 m entre elas. Utilizou-se um total de 24 caixas, considerando cada caixa uma parcela, sendo plantadas três secções de caule de $P$. lapathifolium por parcela. Quando as plantas atingiram o pleno desenvolvimento vegetativo, com altura média de $80 \mathrm{~cm}$, aproximadamente 90 dias após o plantio, foi feita a aplicação dos herbicidas.

Os tratamentos foram 2,4-D amina (U-46 D FLUÍD) a 720 e 1440 g e.a. ha-1; diquat (REWARD) a $480 \mathrm{~g}^{\text {i.a ha }} \mathrm{h}^{-1}$; glyphosate (RODEO) a 2.400 e 3.360 g e.a. ha h $^{-1}+0,5 \%$ v/v de Aterbane; e imazapyr (ARSENAL) a 250 e 500 g e.a. ha-1 . No momento da aplicação de cada herbicida, as demais parcelas foram cobertas com um plástico impermeável, com a finalidade de evitar a ação de uma possível deriva. O delineamento experimental adotado foi o inteiramente casualizado, com três repetições.

A aplicação foi realizada no dia 10/4/2001, utilizando-se um pulverizador costal pressurizado a $\mathrm{CO}_{2}$, equipado com uma barra 
contendo dois bicos de jato plano do tipo Teejet XR 110.02 VS, a pressão constante de 2,0 bars. Estabeleceu-se uma velocidade de deslocamento de $1 \mathrm{~m} \mathrm{~s}^{-1}$, o que proporcionou consumo de calda de $200 \mathrm{~L} \mathrm{ha}^{-1}$. Durante o período de aplicação encontrava-se uma condição de alta luminosidade, em que a temperatura e a umidade relativa do ar variaram entre 28 a $30{ }^{\circ} \mathrm{C}$ e 65 a $62 \%$, respectivamente para o início e final da aplicação.

Foram realizadas avaliações visuais de fitotoxicidade aos $2,4,6,8,10,13,18,28 \mathrm{e}$ 46 dias após a aplicação (DAA), atribuindo-se notas percentuais segundo uma escala, na qual zero correspondeu a nenhum sintoma de injúria e cem à morte da planta.

Os resultados foram submetidos à análise de variância pelo teste $F$, sendo suas médias comparadas pelo teste $t$ a $5 \%$ de probabilidade.

\section{RESULTADOS E DISCUSSÃO}

Observa-se na Tabela 1 que os sintomas provocados pelo herbicida diquat ocorreram de forma rápida e severa; aos $2 \mathrm{DAA}$, foi observado um controle da ordem de $61,7 \%$, que evoluiu para $97,7 \%$ aos 6 DAA. Entretanto, a partir dos 13 DAA as plantas de $P$. lapathifolium apresentaram rebrotas, recuperando-se totalmente aos 46 DAA.
Almeida \& Rodrigues (1998) comentam que o diquat é um herbicida de contato que, quando aplicado em alta luminosidade, pode provocar a morte de plantas sensiveis 24 horas após a aplicação. Por outro lado, Oliveira Jr. \& Costantin (2001) afirmam que a ação rápida deste composto pode prejudicar sua translocação e distribuição na planta, comprometendo sua eficácia no controle de algumas espécies. Dessa maneira, as altas taxas de fitointoxicação proporcionadas inicialmente por este herbicida devem ter prejudicado o controle das plantas de $P$. lapathifolium no decorrer do período de estudo.

As plantas tratadas com 2,4-D expressaram seu grau máximo de toxicidade aos 13 DAA e não ocorreram diferenças entre as doses aplicadas. A partir desse período as plantas começaram a se recuperar, e aquelas tratadas com a menor dose (720 g e.a. ha-1) recuperaram-se mais rapidamente. De maneira geral, plantas suscetiveis a esse tipo de herbicida são eliminadas de três a cinco semanas após a aplicação (Oliveira Jr. \& Constantin, 2001). Verifica-se que a recuperação das plantas iniciou-se aproximadamente duas semanas após a pulverização, evidenciando assim a ineficiência desse produto no controle de $P$. lapathifolium, considerando as doses utilizadas.

Tabela 1 - Porcentagens de controle de Polygonum lapathifolium em diferentes períodos de avaliação após a aplicação de diversos herbicidas. Botucatu-SP, 2001

\begin{tabular}{|c|c|c|c|c|c|c|c|c|c|c|}
\hline \multirow{2}{*}{ Tratamento } & \multirow{2}{*}{$\frac{\text { Dose }}{\left({\left.\text { g e.a. } \text { ha }^{-1}\right)}^{-}\right.}$} & \multicolumn{9}{|c|}{ Dias Após Aplicação } \\
\hline & & 2 & 4 & 6 & 8 & 10 & 13 & 18 & 28 & 46 \\
\hline 1. 2,4-D & 720 & $31,0 \mathrm{c}$ & $36,7 b$ & $72,0 \mathrm{~b}$ & $73,7 \mathrm{~b}$ & $75,7 \mathrm{~b}$ & $77,0 \mathrm{~b}$ & $71,0 \mathrm{~b}$ & $32,7 \mathrm{c}$ & $12,7 \mathrm{c}$ \\
\hline 2. $2,4-\mathrm{D}$ & 1.440 & $36,0 \mathrm{~b}$ & $41,7 b$ & $72,7 \mathrm{~b}$ & $73,3 \mathrm{~b}$ & $74,7 \mathrm{~b}$ & $76,7 \mathrm{bc}$ & $70,0 \mathrm{bc}$ & $43,3 \mathrm{~b}$ & $28,3 \mathrm{~b}$ \\
\hline 3. diquat $^{-1}$ & 480 & $61,7 \mathrm{a}$ & $91,7 \mathrm{a}$ & $97,7 \mathrm{a}$ & $97,7 \mathrm{a}$ & $95,7 \mathrm{a}$ & $87,7 \mathrm{ab}$ & $61,0 \mathrm{c}$ & $22,0 \mathrm{~d}$ & $0,0 \mathrm{~d}$ \\
\hline 4. glyphosate & 2.400 & $0,0 \mathrm{~d}$ & $0,0 \mathrm{c}$ & $5,0 \mathrm{~d}$ & $26,7 \mathrm{c}$ & $69,0 \mathrm{bc}$ & $83,3 \mathrm{ab}$ & $97,0 \mathrm{a}$ & $99,0 \mathrm{a}$ & $100,0 \mathrm{a}$ \\
\hline 5. glyphosate & 3.360 & $0,0 \mathrm{~d}$ & $0,0 \mathrm{c}$ & $11,3 \mathrm{c}$ & $31,7 \mathrm{c}$ & $66,7 \mathrm{c}$ & $92,7 \mathrm{a}$ & $97,0 \mathrm{a}$ & $98,7 \mathrm{a}$ & $100,0 \mathrm{a}$ \\
\hline 6. imazapyr & 250 & $0,0 \mathrm{~d}$ & $0,0 \mathrm{c}$ & $0,0 \mathrm{~d}$ & $2,7 \mathrm{~d}$ & $4,0 \mathrm{~d}$ & $10,0 \mathrm{c}$ & $11,0 \mathrm{~d}$ & $4,0 \mathrm{e}$ & $7,3 \mathrm{c}$ \\
\hline 7. imazapyr & 500 & $0,0 \mathrm{~d}$ & $0,0 \mathrm{c}$ & $0,0 \mathrm{~d}$ & $1,0 \mathrm{~d}$ & $1,7 \mathrm{~d}$ & $7,0 \mathrm{c}$ & 2,7 ed & $6,7 \mathrm{e}$ & $9,3 \mathrm{c}$ \\
\hline 8. testemunha & - & - & - & - & - & - & - & - & - & - \\
\hline $\mathrm{F}$ tratamento & & $562,3 * *$ & $391,1 * *$ & $454,3 * *$ & $96,0 * *$ & $209,2 * *$ & $146,2 * *$ & $168,7 * *$ & $154,5^{* *}$ & $245,4 * *$ \\
\hline CV $(\%)$ & & 10,8 & 13,8 & 10,3 & 17,6 & 9,8 & 10,7 & 10,6 & 14,6 & 14,7 \\
\hline DMS & & 3,02 & 5,08 & 5,77 & 11,75 & 8,19 & 10,17 & 9,41 & 9,74 & 8,14 \\
\hline
\end{tabular}

1/ dose expressa em ingrediente ativo; ** significativo a $1 \%$ de probabilidade. Médias seguidas de mesma letra, na coluna, não diferem estatisticamente entre si pelo teste $\mathrm{t}(\mathrm{P}>0,05)$. 
Estudos com a aplicação isolada de 2,4-D sobre $P$. lapathifolium não foram encontrados. Natal \& Svetov (1973) citam esta espécie como resistente a este herbicida, porém estes mesmos pesquisadores conseguiram reduções de 92 a 97\% em populações desta planta quando aplicaram a mistura com dicamba.

Os sintomas provocados pelo herbicida glyphosate nas plantas de $P$. lapathifolium iniciaram-se a partir dos 6 DAA, evoluindo gradativamente e de forma semelhante entre as doses aplicadas; ao final do estudo, aos 46 DAA, as plantas encontravam-se mortas.

Whatley et al. (1984) comentam que a dose de 208,5 g e.a. ha-1 de glyphosate proporcionou controle de $86 \%$ desta espécie, quando tratada em estágio inicial de desenvolvimento. A dose utilizada por estes pesquisadores foi baixa quando comparada com as utilizadas neste trabalho, o que conduz à hipótese de utilização de doses menores. Entretanto, dois fatores justificam a utilização das doses testadas: o primeiro é o estágio de aplicação, no qual se trabalhou com plantas adultas, sendo esta a condição mais comum em ambiente aquático; e o segundo refere-se ao melhor nível de controle obtido - $100 \%$ neste caso.

As plantas tratadas com o herbicida imazapyr apresentaram leves sintomas de intoxicação a partir dos 8 DAA, com o seu maior efeito observado aos $18 \mathrm{DAA}$. As injúrias provocadas por este herbicida foram caracterizadas por leve amarelecimento foliar com alguns pontos de necrose, porém estes sintomas não evoluíram - ao final do experimento, foram observadas pequenas injúrias somente nas folhas mais velhas. Não houve diferença entre as doses testadas, sendo ambas ineficientes no controle desta espécie.

\section{LITERATURA CITADA}

ALMEIDA, F. S.; RODRIGUES, B. N. Guia de herbicidas. Londrina: 1998. p. 648.

DEUBER, R. Ciências das plantas infestantes - Manejo. Campinas: 1997. 285 p.

LORENZI, H. Plantas daninhas do Brasil. 3.ed. Nova Odessa: Instituto Plantarum. 1992. p. 515.

MARCONDES, D. A. S.; TANAKA, R. H. Plantas aquáticas nos reservatórios das usinas hidrelétricas da CESP. In: CONGRESSO BRASILEIRO DAS PLANTAS DANINHAS, 21., 1997, Caxambu. Plantas Aquáticas. Caxambu: SBCPD, 1997. p. 2-4.

MARTINS, D. Controle químico de plantas daninhas aquáticas. In: WORKSHOP - CONTROLE DE PLANTAS AQUÁTICAS, 1998, Brasília. Resumos... Brasília: IBAMA 1998. p. 30-31.

OLIVEIRA JR., R. S.; CONSTANTIN J. Mecanismo de ação de herbicidas. In: Plantas daninhas e seu manejo. Guaíba: Agropecuária, 2001. p. 207-260.

PITELI, R. A. Macrófitas aquáticas no Brasil, na condição de problemática. In: WORKSHOP - CONTROLE DE PLANTAS AQUÁTICAS, 1998, Brasília. Resumos... Brasília: IBAMA. 1998. p. 12-15.

TANAKA, R. H. Prejuízos provocados pelas plantas aquáticas. In: WORKSHOP - CONTROLE DE PLANTAS AQUÁTICAS, 1998, Brasília. Resumos... Brasília: IBAMA 1998. p. 36-38.

WHATLEY,T. L.; SANDBERG, C. L.; WU, C. H. Annual weed control on stale seedbeds with glyphosate. In: SOUTHERN WEED SCIENCE SOCIETY, 37., Illinois. CAB Abstracts, 1984-1986.

NATAL, O. B.; SVETOV, V. G. Herbicides for oats. Zashchita-Rastenii, v. 27, n. 23, CAB Abstratct, 19721975. 1984. p. 53-56. 